\title{
Altitude, Orthocenter of a Triangle and Triangulation
}

\author{
Roland Coghetto \\ Rue de la Brasserie 5 \\ 7100 La Louvière, Belgium
}

\begin{abstract}
Summary. We introduce the altitudes of a triangle (the cevians perpendicular to the opposite sides). Using the generalized Ceva's Theorem, we prove the existence and uniqueness of the orthocenter of a triangle [7. Finally, we formalize in Mizar [1] some formulas 2] to calculate distance using triangulation.
\end{abstract}

MSC: 51M04 03B35

Keywords: Euclidean geometry; trigonometry; altitude; orthocenter; triangulation; distance

MML identifier: EUCLID13, version: 8.1.04 5.36.1267

\section{Preliminaries}

From now on $n$ denotes a natural number, $i$ denotes an integer, $r, s, t$ denote real numbers, $A_{1}, B_{1}, C_{1}, D_{1}$ denote points of $\mathcal{E}_{\mathrm{T}}^{n}, L_{1}, L_{2}$ denote elements of Lines $\left(\mathcal{R}^{n}\right)$, and $A, B, C$ denote points of $\mathcal{E}_{\mathrm{T}}^{2}$.

Now we state the propositions:

(1) If $0<i \cdot r<r$, then $i=1$.

(2) Let us consider an integer $i$. If $\frac{-3}{2}<i<\frac{1}{2}$, then $i=0$ or $i=-1$.

(3) Suppose $r$ is not zero and $s$ is not zero and $t$ is not zero. Then $\left(\frac{-r}{-s}\right)$. $\left(\frac{-t}{-r}\right) \cdot\left(\frac{-s}{-t}\right)=1$.

(4) If $0<r<2 \cdot \pi$, then $\sin \left(\frac{r}{2}\right) \neq 0$. The theorem is a consequence of (1).

(5) If $-2 \cdot \pi<r<0$, then $\sin \left(\frac{r}{2}\right) \neq 0$. The theorem is a consequence of (4).

(6) $\tan (2 \cdot \pi-r)=-\tan r$.

(7) If $A_{1} \in \operatorname{Line}\left(B_{1}, C_{1}\right)$ and $A_{1} \neq C_{1}$, then $\operatorname{Line}\left(B_{1}, C_{1}\right)=\operatorname{Line}\left(A_{1}, C_{1}\right)$. 
(8) If $A_{1} \neq C_{1}$ and $A_{1} \in \operatorname{Line}\left(B_{1}, C_{1}\right)$, then $B_{1} \in \operatorname{Line}\left(A_{1}, C_{1}\right)$.

(9) Suppose $A_{1} \neq B_{1}$ and $A_{1} \neq C_{1}$ and $\left|\left(A_{1}-B_{1}, A_{1}-C_{1}\right)\right|=0$ and $L_{1}=\operatorname{Line}\left(A_{1}, B_{1}\right)$ and $L_{2}=\operatorname{Line}\left(A_{1}, C_{1}\right)$. Then $L_{1} \perp L_{2}$.

(10) If $B_{1} \neq C_{1}$ and $\left|\left(B_{1}-A_{1}, B_{1}-C_{1}\right)\right|=0$, then $A_{1} \neq C_{1}$.

(11) $\left|\left(A_{1}-B_{1}, A_{1}-C_{1}\right)\right|=\left|\left(B_{1}-A_{1}, C_{1}-A_{1}\right)\right|$.

(12) Suppose $B_{1} \neq C_{1}$ and $r=-\left(\frac{\left|\left(B_{1}, C_{1}\right)\right|-\left|\left(C_{1}, C_{1}\right)\right|-\left|\left(A_{1}, B_{1}\right)\right|+\left|\left(A_{1}, C_{1}\right)\right|}{\left|\left(B_{1}-C_{1}, B_{1}-C_{1}\right)\right|}\right)$ and $D_{1}=r \cdot B_{1}+(1-r) \cdot C_{1}$. Then $\left|\left(D_{1}-A_{1}, D_{1}-C_{1}\right)\right|=0$.

(13) If $A_{1} \neq B_{1}$ and $C_{1}=r \cdot A_{1}+(1-r) \cdot B_{1}$ and $C_{1}=B_{1}$, then $r=0$.

(14) (i) $\left|\left(B_{1}, C_{1}\right)\right|-\left|\left(C_{1}, C_{1}\right)\right|-\left|\left(A_{1}, B_{1}\right)\right|+\left|\left(A_{1}, C_{1}\right)\right|=\left|\left(C_{1}-A_{1}, B_{1}-C_{1}\right)\right|$, and

(ii) $\left|\left(B_{1}-C_{1}, B_{1}-C_{1}\right)\right|+\left|\left(C_{1}-A_{1}, B_{1}-C_{1}\right)\right|=\left|\left(B_{1}-C_{1}, B_{1}-A_{1}\right)\right|$.

(15) $\left|\left(A_{1}-B_{1}, A_{1}-C_{1}\right)\right|=-\left|\left(A_{1}-B_{1}, C_{1}-A_{1}\right)\right|$.

(16) $\left|\left(B_{1}-A_{1}, C_{1}-A_{1}\right)\right|=\left|\left(A_{1}-B_{1}, A_{1}-C_{1}\right)\right|$.

(17) $\left|\left(B_{1}-A_{1}, C_{1}-A_{1}\right)\right|=-\left|\left(B_{1}-A_{1}, A_{1}-C_{1}\right)\right|$. The theorem is a consequence of (16) and (15).

(18) Suppose $B_{1} \neq C_{1}$ and $C_{1} \neq A_{1}$ and $A_{1} \neq B_{1}$ and $\left|\left(C_{1}-A_{1}, B_{1}-C_{1}\right)\right|$ is not zero and $\left|\left(B_{1}-C_{1}, A_{1}-B_{1}\right)\right|$ is not zero and $\mid\left(C_{1}-A_{1}, A_{1}-\right.$ $\left.B_{1}\right) \mid$ is not zero and $r=-\left(\frac{\left|\left(B_{1}, C_{1}\right)\right|-\left|\left(C_{1}, C_{1}\right)\right|-\left|\left(A_{1}, B_{1}\right)\right|+\left|\left(A_{1}, C_{1}\right)\right|}{\left|\left(B_{1}-C_{1}, B_{1}-C_{1}\right)\right|}\right)$ and $s=$ $-\left(\frac{\left|\left(C_{1}, A_{1}\right)\right|-\left|\left(A_{1}, A_{1}\right)\right|-\left|\left(B_{1}, C_{1}\right)\right|+\left|\left(B_{1}, A_{1}\right)\right|}{\left|\left(C_{1}-A_{1}, C_{1}-A_{1}\right)\right|}\right)$ and

$t=-\left(\frac{\left|\left(A_{1}, B_{1}\right)\right|-\left|\left(B_{1}, B_{1}\right)\right|-\left|\left(C_{1}, A_{1}\right)\right|+\left|\left(C_{1}, B_{1}\right)\right|}{\left|\left(A_{1}-B_{1}, A_{1}-B_{1}\right)\right|}\right)$ Then $\frac{\left(\frac{\left(\frac{r}{1-r}\right) \cdot s}{1-s}\right) \cdot t}{1-t}=1$. The theorem is a consequence of (14), (15), and (3).

(19) If $C_{1}=r \cdot A_{1}+(1-r) \cdot B_{1}$ and $r=1$, then $C_{1}=A_{1}$.

(20) If $C_{1}=r \cdot A_{1}+(1-r) \cdot B_{1}$ and $r=0$, then $C_{1}=B_{1}$.

(21) If $\left|\left(B_{1}-C_{1}, B_{1}-C_{1}\right)\right|=-\left|\left(C_{1}-A_{1}, B_{1}-C_{1}\right)\right|$, then $\mid\left(B_{1}-C_{1}, A_{1}-\right.$ $\left.B_{1}\right) \mid=0$. The theorem is a consequence of (15).

(22) Suppose $B_{1} \neq C_{1}$ and $r=-\left(\frac{\left|\left(B_{1}, C_{1}\right)\right|-\left|\left(C_{1}, C_{1}\right)\right|-\left|\left(A_{1}, B_{1}\right)\right|+\left|\left(A_{1}, C_{1}\right)\right|}{\left|\left(B_{1}-C_{1}, B_{1}-C_{1}\right)\right|}\right)$ and $r=1$. Then $\left|\left(B_{1}-C_{1}, A_{1}-B_{1}\right)\right|=0$. The theorem is a consequence of (14) and (21).

(23) If $A \neq B$ and $A \neq C$, then $|A-B|+|A-C| \neq 0$.

(24) If $A, B, C$ form a triangle, then $A \notin \operatorname{Line}(B, C)$.

(25) If $A \neq B$ and $B \neq C$ and $|(B-A, B-C)|=0$, then $\measuredangle(A, B, C)=\frac{\pi}{2}$ or $\measuredangle(A, B, C)=\left(\frac{3}{2}\right) \cdot \pi$.

(26) If $A, B, C$ form a triangle, then $\sin \left(\frac{\measuredangle(A, B, C)}{2}\right)>0$.

(27) If $\measuredangle(B, A, C) \neq \measuredangle(C, B, A)$, then $\sin \left(\frac{\measuredangle(B, A, C)-\measuredangle(C, B, A)}{2}\right) \neq 0$. The theorem is a consequence of (5) and (4). 
(28) If $A, B, C$ form a triangle, then $\sin \measuredangle(A, B, C) \neq 0$.

Let us assume that $A, C, B$ form a triangle and $\measuredangle(A, C, B)<\pi$. Now we state the propositions:

(29) $\measuredangle(A, C, B)=\pi-(\measuredangle(C, B, A)+\measuredangle(B, A, C))$.

(30) $\measuredangle(B, A, C)+\measuredangle(C, B, A)=\pi-\measuredangle(A, C, B)$. The theorem is a consequence of $(29)$.

Let us assume that $A, B, C$ form a triangle. Now we state the propositions:

(31) $\measuredangle(B, A, C)-\measuredangle(C, B, A) \neq \pi$.

(32) $\measuredangle(B, A, C)-\measuredangle(C, B, A) \neq-\pi$.

Let us assume that $A, B, C$ form a triangle. Now we state the propositions:

(33) $\quad(-2) \cdot \pi<\measuredangle(B, A, C)-\measuredangle(C, B, A)<2 \cdot \pi$.

(34) $-\pi<\frac{\measuredangle(B, A, C)-\measuredangle(C, B, A)}{2}<\pi$. The theorem is a consequence of (33).

Let us assume that $A, B, C$ form a triangle and $\measuredangle(B, A, C)<\pi$. Now we state the propositions:

(35) $-\pi<\measuredangle(B, A, C)-\measuredangle(C, B, A)<\pi$.

(36) $-\left(\frac{\pi}{2}\right)<\frac{\measuredangle(B, A, C)-\measuredangle(C, B, A)}{2}<\frac{\pi}{2}$. The theorem is a consequence of $(35)$.

\section{ORTHOCENTER}

From now on $D$ denotes a point of $\mathcal{E}_{\mathrm{T}}^{2}$ and $a, b, c, d$ denote real numbers.

Let $A, B, C$ be points of $\mathcal{E}_{\mathrm{T}}^{2}$. Assume $B \neq C$. The functor Altit $\triangle(A, B, C)$ yielding an element of $\operatorname{Lines}\left(\mathcal{R}^{2}\right)$ is defined by

(Def. 1) there exist elements $L_{1}, L_{2}$ of $\operatorname{Lines}\left(\mathcal{R}^{2}\right)$ such that $i t=L_{1}$ and $L_{2}=$ Line $(B, C)$ and $A \in L_{1}$ and $L_{1} \perp L_{2}$.

Let us assume that $B \neq C$. Now we state the propositions:

(37) $A \in$ Altit $\triangle(A, B, C)$.

(38) Altit $\triangle(A, B, C)$ is a line.

(39) Altit $\triangle(A, B, C)=$ Altit $\triangle(A, C, B)$.

Now we state the propositions:

(40) If $B \neq C$ and $D \in$ Altit $\triangle(A, B, C)$, then

Altit $\triangle(D, B, C)=$ Altit $\triangle(A, B, C)$.

(41) If $B \neq C$ and $D \in \operatorname{Line}(B, C)$ and $D \neq C$, then Altit $\triangle(A, B, C)=$ Altit $\triangle(A, D, C)$. The theorem is a consequence of $(7)$.

Let $A, B, C$ be points of $\mathcal{E}_{\mathrm{T}}^{2}$. Assume $B \neq C$. The functor FootAltit $\triangle(A, B, C)$ yielding a point of $\mathcal{E}_{\mathrm{T}}^{2}$ is defined by 
(Def. 2) there exists a point $P$ of $\mathcal{E}_{\mathrm{T}}^{2}$ such that it $=P$ and Altit $\triangle(A, B, C) \cap$ $\operatorname{Line}(B, C)=\{P\}$.

Let us assume that $B \neq C$. Now we state the propositions:

(42) FootAltit $\triangle(A, B, C)=$ FootAltit $\triangle(A, C, B)$. The theorem is a consequence of (39).

(43) (i) FootAltit $\triangle(A, B, C) \in \operatorname{Line}(B, C)$, and

(ii) FootAltit $\triangle(A, B, C) \in$ Altit $\triangle(A, B, C)$.

Now we state the propositions:

(44) If $B \neq C$ and $A \notin \operatorname{Line}(B, C)$, then Altit $\triangle(A, B, C)=$ Line $(A$, FootAltit $\triangle(A, B, C))$. The theorem is a consequence of (43).

(45) If $B \neq C$ and $A \in \operatorname{Line}(B, C)$, then FootAltit $\triangle(A, B, C)=A$.

(46) If $B \neq C$ and FootAltit $\triangle(A, B, C)=A$, then $A \in \operatorname{Line}(B, C)$.

Let us assume that $B \neq C$. Now we state the propositions:

(47) $\mid(A-$ FootAltit $\triangle(A, B, C), B-C) \mid=0$. The theorem is a consequence of (44) and (45).

(48) $\mid(A$-FootAltit $\triangle(A, B, C), B$-FootAltit $\triangle(A, B, C)) \mid=0$. The theorem is a consequence of (43), (44), and (45).

(49) $\mid(A$-FootAltit $\triangle(A, B, C), C$ - FootAltit $\triangle(A, B, C)) \mid=0$. The theorem is a consequence of (42) and (48).

Now we state the propositions:

(50) If $B \neq C$ and $B=$ FootAltit $\triangle(A, B, C)$, then $|(B-A, B-C)|=0$. The theorem is a consequence of (49), (11), and (43).

(51) If $B \neq C$ and $D \in \operatorname{Line}(B, C)$ and $D \neq C$, then FootAltit $\triangle(A, B, C)=$ FootAltit $\triangle(A, D, C)$. The theorem is a consequence of (7) and (41).

(52) If $B \neq C$ and $|(B-A, B-C)|=0$, then $B=$ FootAltit $\triangle(A, B, C)$. The theorem is a consequence of (9) and (45).

(53) If $B \neq C$ and $B \neq A$ and $\measuredangle(A, B, C)=\frac{\pi}{2}$, then FootAltit $\triangle(A, B, C)=$ $B$. The theorem is a consequence of (11) and (52).

(54) If $A, B, C$ form a triangle, then $A \neq$ FootAltit $\triangle(A, B, C)$. The theorem is a consequence of (43).

(55) If $A, B, C$ form a triangle and $|(B-A, B-C)| \neq 0$, then

FootAltit $\triangle(A, B, C), B, A$ form a triangle.

Proof: Set $p=$ FootAltit $\triangle(A, B, C)$. Consider $P$ being a point of $\mathcal{E}_{\mathrm{T}}^{2}$ such that FootAltit $\triangle(A, B, C)=P$ and Altit $\triangle(A, B, C) \cap \operatorname{Line}(B, C)=\{P\}$. Consider $L_{1}, L_{2}$ being elements of Lines $\left(\mathcal{R}^{2}\right)$ such that Altit $\triangle(A, B, C)=$ $L_{1}$ and $L_{2}=\operatorname{Line}(B, C)$ and $A \in L_{1}$ and $L_{1} \perp L_{2} . P \neq B . p \neq A$. $p, B, A$ are mutually different. $P \in \operatorname{Line}(B, C) . B, C \in \operatorname{Line}(B, P) . \measuredangle(p, B, A) \neq \pi$ 
by [11, (11)], [12, (12)], (50), (8). $\measuredangle(B, A, p) \neq \pi$ by [11, (11)], [12, (12)]. $\measuredangle(A, p, B) \neq \pi$ by [11, (11)], [12, (12)], (8), (54).

Let $A, B, C$ be points of $\mathcal{E}_{\mathrm{T}}^{2}$. Assume $B \neq C$. The functor $\mid$ Altit $\triangle(A, B, C) \mid$ yielding a real number is defined by the term

(Def. 3) $\mid A$ - FootAltit $\triangle(A, B, C) \mid$.

Let us assume that $B \neq C$. Now we state the propositions:

(56) $0 \leqslant \mid$ Altit $\triangle(A, B, C) \mid$.

(57) $\mid$ Altit $\triangle(A, B, C)|=|$ Altit $\triangle(A, C, B) \mid$. The theorem is a consequence of (42).

Now we state the propositions:

(58) If $B \neq C$ and $|(B-A, B-C)|=0$, then $\mid$ FootAltit $\triangle(A, B, C)-A \mid=$ $|A-B|$. The theorem is a consequence of (52).

(59) Suppose $B \neq C$ and $r=-\left(\frac{|(B, C)|-|(C, C)|-|(A, B)|+|(A, C)|}{|(B-C, B-C)|}\right)$ and $D=r \cdot B+$ $(1-r) \cdot C$ and $D \neq C$. Then $D=$ FootAltit $\triangle(A, B, C)$.

Proof: $|(D-A, D-C)|=0 . D=$ FootAltit $\triangle(A, D, C) . D \in \operatorname{Line}(B, C)$ by [6, (4)].

(60) Suppose $B \neq C$ and $r=-\left(\frac{|(B, C)|-|(C, C)|-|(A, B)|+|(A, C)|}{|(B-C, B-C)|}\right)$ and $D=r \cdot B+$ $(1-r) \cdot C$ and $D=C$. Then $C=$ FootAltit $\triangle(A, B, C)$. The theorem is a consequence of (13), (14), (15), (52), and (42).

(61) Suppose $A, B, C$ form a triangle and $|(C-A, B-C)|$ is not zero and $|(B-C, A-B)|$ is not zero and $|(C-A, A-B)|$ is not zero. Then Line $(A$, FootAltit $\triangle(A, B, C))$, Line $(C$, FootAltit $\triangle(C, A, B))$,

Line $(B$, FootAltit $\triangle(B, C, A))$ are concurrent. The theorem is a consequence of (60), (17), (47), (59), (18), and (22).

(62) If $A, B, C$ form a triangle and $|(C-A, B-C)|$ is zero, then FootAltit $\triangle(A, B, C)=C$ and FootAltit $\triangle(B, C, A)=C$. The theorem is a consequence of (15), (52), and (42).

(63) Suppose $A, B, C$ form a triangle and $C \in$ Altit $\triangle(A, B, C)$ and $C \in$ Altit $\triangle(B, C, A)$. Then Altit $\triangle(A, B, C) \cap$ Altit $\triangle(B, C, A)$ is a point.

Proof: Consider $L_{1}, L_{2}$ being elements of Lines $\left(\mathcal{R}^{2}\right)$ such that Altit $\triangle(A, B, C)=L_{1}$ and $L_{2}=\operatorname{Line}(B, C)$ and $A \in L_{1}$ and $L_{1} \perp L_{2}$. Consider $L_{3}, L_{4}$ being elements of Lines $\left(\mathcal{R}^{2}\right)$ such that Altit $\triangle(B, C, A)=$ $L_{3}$ and $L_{4}=\operatorname{Line}(C, A)$ and $B \in L_{3}$ and $L_{3} \perp L_{4} . L_{1} \nVdash L_{3}$ by [9, (41)], [6, (16)], [8, (108)], [12, (13)]. $L_{1}$ is not a point and $L_{3}$ is not a point.

(64) Suppose $B, C, A$ form a triangle and $C \in$ Altit $\triangle(B, C, A)$ and $C \in$ Altit $\triangle(C, A, B)$. Then Altit $\triangle(B, C, A) \cap$ Altit $\triangle(C, A, B)$ is a point. Proof: Consider $L_{1}, L_{2}$ being elements of Lines $\left(\mathcal{R}^{2}\right)$ such that 
Altit $\triangle(B, C, A)=L_{1}$ and $L_{2}=\operatorname{Line}(C, A)$ and $B \in L_{1}$ and $L_{1} \perp L_{2}$. Consider $L_{3}, L_{4}$ being elements of Lines $\left(\mathcal{R}^{2}\right)$ such that Altit $\triangle(C, A, B)=$ $L_{3}$ and $L_{4}=\operatorname{Line}(A, B)$ and $C \in L_{3}$ and $L_{3} \perp L_{4} . L_{1} \nVdash L_{3}$ by [8, (71), (111)], [6, (16)], [9, (41)]. $L_{1}$ is not a point and $L_{3}$ is not a point.

(65) Suppose $C, A, B$ form a triangle and $C \in$ Altit $\triangle(C, A, B)$ and $C \in$ Altit $\triangle(A, B, C)$. Then Altit $\triangle(C, A, B) \cap$ Altit $\triangle(A, B, C)$ is a point.

Proof: Consider $L_{1}, L_{2}$ being elements of Lines $\left(\mathcal{R}^{2}\right)$ such that Altit $\triangle(C, A, B)=L_{1}$ and $L_{2}=\operatorname{Line}(A, B)$ and $C \in L_{1}$ and $L_{1} \perp L_{2}$. Consider $L_{3}, L_{4}$ being elements of Lines $\left(\mathcal{R}^{2}\right)$ such that Altit $\triangle(A, B, C)=$ $L_{3}$ and $L_{4}=\operatorname{Line}(B, C)$ and $A \in L_{3}$ and $L_{3} \perp L_{4} . L_{1} \nVdash L_{3}$ by [8, (71), (111)], [6, (16)], [9, (41)]. $L_{1}$ is not a point and $L_{3}$ is not a point.

(66) Suppose $A, B, C$ form a triangle and $|(C-A, B-C)|=0$. Then

(i) Altit $\triangle(A, B, C) \cap$ Altit $\triangle(B, C, A)=\{C\}$, and

(ii) Altit $\triangle(B, C, A) \cap$ Altit $\triangle(C, A, B)=\{C\}$, and

(iii) Altit $\triangle(C, A, B) \cap$ Altit $\triangle(A, B, C)=\{C\}$.

Proof: $A \notin \operatorname{Line}(B, C)$ and $B \notin \operatorname{Line}(C, A)$. FootAltit $\triangle(A, B, C)=$ $C$ and FootAltit $\triangle(B, C, A)=C$. Altit $\triangle(A, B, C)=\operatorname{Line}(A, C)$ and Altit $\triangle(B, C, A)=\operatorname{Line}(B, C) . C \in$ Altit $\triangle(C, A, B)$. Altit $\triangle(A, B, C) \cap$ Altit $\triangle(B, C, A)=\{C\}$ by [6], (22)], (63). Altit $\triangle(B, C, A) \cap$ Altit $\triangle(C, A, B)$ $=\{C\}$ by [12, (15)], (37), (64), [6, (22)]. Altit $\triangle(C, A, B) \cap$ Altit $\triangle(A, B, C)$ $=\{C\}$ by [12, (15)], (37), (65), [6, (22)].

(67) Suppose $A, B, C$ form a triangle. Then there exists a point $P$ of $\mathcal{E}_{\mathrm{T}}^{2}$ such that

(i) Altit $\triangle(A, B, C) \cap$ Altit $\triangle(B, C, A)=\{P\}$, and

(ii) Altit $\triangle(B, C, A) \cap$ Altit $\triangle(C, A, B)=\{P\}$, and

(iii) Altit $\triangle(C, A, B) \cap$ Altit $\triangle(A, B, C)=\{P\}$.

The theorem is a consequence of (66), (61), (24), (44), and (38).

Let $A, B, C$ be points of $\mathcal{E}_{\mathrm{T}}^{2}$. Assume $A, B, C$ form a triangle. The functor Orthocenter $\triangle(A, B, C)$ yielding a point of $\mathcal{E}_{\mathrm{T}}^{2}$ is defined by

(Def. 4) Altit $\triangle(A, B, C) \cap$ Altit $\triangle(B, C, A)=\{$ it $\}$ and Altit $\triangle(B, C, A) \cap$ Altit $\triangle(C, A, B)=\{i t\}$ and Altit $\triangle(C, A, B) \cap$ Altit $\triangle(A, B, C)=\{i t\}$.

\section{Triangulation}

Let us assume that $B \neq A$. Now we state the propositions:

(68) $\quad(\sin \measuredangle(B, A, C)+\sin \measuredangle(C, B, A)) \cdot(|C-B|-|C-A|)=(\sin \measuredangle(B, A, C)-$ $\sin \measuredangle(C, B, A)) \cdot(|C-B|+|C-A|)$. 
(69) $\sin \left(\frac{\measuredangle(B, A, C)+\measuredangle(C, B, A)}{2}\right) \cdot \cos \left(\frac{\measuredangle(B, A, C)-\measuredangle(C, B, A)}{2}\right) \cdot(|C-B|-|C-A|)=$ $\sin \left(\frac{\measuredangle(B, A, C)-\measuredangle(C, B, A)}{2}\right) \cdot \cos \left(\frac{\measuredangle(B, A, C)+\measuredangle(C, B, A)}{2}\right) \cdot(|C-B|+|C-A|)$. The theorem is a consequence of (68).

Now we state the proposition:

(70) Suppose $A, B, C$ form a triangle and $\measuredangle(B, A, C)-\measuredangle(C, B, A) \neq \pi$ and $\measuredangle(B, A, C)-\measuredangle(C, B, A) \neq-\pi$. Then $\cos \left(\frac{\measuredangle(B, A, C)-\measuredangle(C, B, A)}{2}\right) \neq 0$. The theorem is a consequence of (2).

Let us assume that $A, C, B$ form a triangle and $\measuredangle(A, C, B)<\pi$. Now we state the propositions:

(71) $\tan \left(\frac{\measuredangle(B, A, C)-\measuredangle(C, B, A)}{2}\right)=\cot \left(\frac{\measuredangle(A, C, B)}{2}\right) \cdot\left(\frac{|C-B|-|C-A|}{|C-B|+|C-A|}\right)$.

Proof: $\measuredangle(B, A, C)-\measuredangle(C, B, A) \neq \pi$ and $\measuredangle(B, A, C)-\measuredangle(C, B, A) \neq-\pi$. Set $\alpha=\frac{\measuredangle(B, A, C)-\measuredangle(C, B, A)}{2}$. Set $\beta=\frac{\measuredangle(B, A, C)+\measuredangle(C, B, A)}{2} . \measuredangle(A, C, B)=\pi-$ $(\measuredangle(C, B, A)+\measuredangle(B, A, C))$. Set $\alpha_{1}=\frac{\measuredangle(A, C, B)}{2} \cdot \sin \alpha_{1} \neq 0 .|C-B|+|C-A| \neq$ 0 by [11, (42)]. $\sin \beta \cdot \cos \alpha \cdot(|C-B|-|C-A|)=\sin \alpha \cdot \cos \beta \cdot(|C-B|+$ $|C-A|) \cdot(|C-B|-|C-A|) \cdot \cos \alpha_{1} \cdot 1=(|C-B|+|C-A|) \cdot \sin \alpha_{1} \cdot\left(\frac{\sin \alpha}{\cos \alpha}\right)$.

(72) $\frac{\measuredangle(B, A, C)-\measuredangle(C, B, A)}{2}=\arctan \left(\cot \left(\frac{\measuredangle(A, C, B)}{2}\right) \cdot\left(\frac{|C-B|-|C-A|}{|C-B|+|C-A|}\right)\right)$. The theorem is a consequence of (71) and (36).

(73) $\measuredangle(B, A, C)-\measuredangle(C, B, A)=2 \cdot \arctan \left(\cot \left(\frac{\measuredangle(A, C, B)}{2}\right) \cdot\left(\frac{|C-B|-|C-A|}{|C-B|+|C-A|}\right)\right)$. The theorem is a consequence of (72).

(74) (i) $\measuredangle(B, A, C)=\arctan \left(\cot \left(\frac{\measuredangle(A, C, B)}{2}\right) \cdot\left(\frac{|C-B|-|C-A|}{|C-B|+|C-A|}\right)\right)+\left(\frac{\pi}{2}\right)-\left(\frac{\measuredangle(A, C, B)}{2}\right)$, and

(ii) $\measuredangle(C, B, A)=\left(\frac{\pi}{2}\right)-\left(\frac{\measuredangle(A, C, B)}{2}\right)-\arctan \left(\cot \left(\frac{\measuredangle(A, C, B)}{2}\right) \cdot\left(\frac{|C-B|-|C-A|}{|C-B|+|C-A|}\right)\right)$. The theorem is a consequence of (73) and (30).

(75) $|B-C|=\frac{|A-B| \cdot \sin \measuredangle(B, A, C)}{\sin (\measuredangle(B, A, C)+\measuredangle(C, B, A))}$.

Proof: $|B-C|=\frac{|A-B| \cdot \sin \measuredangle(B, A, C)}{\sin \measuredangle(A, C, B)}$ by [11, (6), (43)], (28). $\measuredangle(A, C, B)=$ $\pi-(\measuredangle(C, B, A)+\measuredangle(B, A, C))$.

(76) $|A-C|=\frac{|A-B| \cdot \sin \measuredangle(C, B, A)}{\sin (\measuredangle(B, A, C)+\measuredangle(C, B, A))}$.

Proof: $|A-C|=\frac{|A-B| \cdot \sin \measuredangle(C, B, A)}{\sin \measuredangle(A, C, B)}$ by [11, (6)], (28). $\measuredangle(A, C, B)=\pi-$ $(\measuredangle(C, B, A)+\measuredangle(B, A, C))$ by $[11,(20)],[10,(47)]$.

Now we state the propositions:

(77) Suppose $A, C, B$ form a triangle and $\measuredangle(C, A, B)=\frac{\pi}{2}$.

Then $\mid$ Altit $\triangle(C, A, B)|=| A-B \mid \cdot \tan \measuredangle(A, B, C)$. The theorem is a consequence of (11) and (58).

(78) Suppose $A, B, C$ form a triangle and $\measuredangle(C, A, B)=\left(\frac{3}{2}\right) \cdot \pi$. 
Then $\mid$ Altit $\triangle(C, A, B)|=| A-B \mid \cdot \tan \measuredangle(C, B, A)$. The theorem is a consequence of (11) and (58).

(79) Suppose $A, C, B$ form a triangle and $|(A-C, A-B)|=0$. Then $\mid$ Altit $\triangle(C, A, B)|=| A-B|\cdot| \tan \measuredangle(A, B, C) \mid$. The theorem is a consequence of (11), (77), (56), (6), and (78).

(80) Suppose $B \neq C$ and FootAltit $\triangle(A, B, C), B, A$ form a triangle. Then

(i) $|A-B| \cdot \sin \measuredangle(A, B$, FootAltit $\triangle(A, B, C))=\mid$ FootAltit $\triangle(A, B, C)-$ $A \mid$, or

(ii) $|A-B| \cdot(-\sin \measuredangle(A, B$, FootAltit $\triangle(A, B, C)))=$ | FootAltit $\triangle(A, B, C)-A \mid$.

The theorem is a consequence of (48).

(81) Suppose $A, B, C$ form a triangle and $|(B-A, B-C)| \neq 0$. Then

(i) $|A-B| \cdot \sin \measuredangle(A, B$, FootAltit $\triangle(A, B, C))=\mid$ FootAltit $\triangle(A, B, C)-$ $A \mid$, or

(ii) $|A-B| \cdot(-\sin \measuredangle(A, B$, FootAltit $\triangle(A, B, C)))=$ | FootAltit $\triangle(A, B, C)-A \mid$.

The theorem is a consequence of (80) and (55).

(82) Suppose $A, C, B$ form a triangle and $\measuredangle(A, C, B)<\pi$ and $\mid(A-C, A-$ $B) \mid \neq 0$. Then $\mid$ Altit $\triangle(C, A, B)|=| A-B|\cdot|\left(\frac{\sin \measuredangle(C, B, A)}{\sin (\measuredangle(B, A, C)+\measuredangle(C, B, A))}\right) \cdot$ $\sin \measuredangle(C, A$, FootAltit $\triangle(C, A, B)) \mid$. The theorem is a consequence of $(76)$, $(55)$, and (80).

(83) Suppose $0<\measuredangle(B, A, D)<\pi$ and $0<\measuredangle(D, A, C)<\pi$ and $D, A, C$ are mutually different and $B, A, D$ are mutually different. Then $\measuredangle(A, C, D)+$ $\measuredangle(D, B, A)=2 \cdot \pi-(\measuredangle(B, A, C)+\measuredangle(A, D, B)+\measuredangle(C, D, A))$.

Proof: $\measuredangle(B, A, D)+\measuredangle(D, A, C)=\measuredangle(B, A, C)$ by [5, (2)], [11, (4)]. $\measuredangle(A, C$, $D)=\pi-(\measuredangle(C, D, A)+\measuredangle(D, A, C))$ by [10, (47)]. $\measuredangle(D, B, A)=\pi-$ $(\measuredangle(A, D, B)+\measuredangle(B, A, D))$ by [10, (47)].

(84) Suppose $A, C, B$ form a triangle and $\measuredangle(A, C, B)<\pi$ and $A, D, B$ form a triangle and $\measuredangle(A, D, B)<\pi$ and $a=\measuredangle(C, B, A)$ and $b=\measuredangle(B, A, C)$ and $c=\measuredangle(D, B, A)$ and $d=\measuredangle(C, A, D)$. Then $|D-C|^{2}=|A-B|^{2}$. $\left(\left(\frac{\sin a}{\sin (a+b)}\right)^{2}+\left(\frac{\sin c}{\sin (b+d+c)}\right)^{2}-2 \cdot\left(\frac{\sin a}{\sin (b+a)}\right) \cdot\left(\frac{\sin c}{\sin (b+d+c)}\right) \cdot \cos d\right)$.

Proof: Set $e=b+d \cdot \sin (e+c)=\sin (\measuredangle(B, A, D)+\measuredangle(D, B, A))$ by [14, (79)].

(85) Suppose $\sin (2 \cdot s) \cdot \cos d=\cos (2 \cdot t)$. Then $(r \cdot \cos s)^{2}+(r \cdot \sin s)^{2}-2$. $(r \cdot \cos s) \cdot(r \cdot \sin s) \cdot \cos d=2 \cdot r^{2} \cdot(\sin t)^{2}$.

(86) Let us consider real numbers $R, \vartheta$. Suppose $D \neq C$ and $0 \leqslant R$ and $A$, $C, B$ form a triangle and $\measuredangle(A, C, B)<\pi$ and $A, D, B$ form a triangle 
and $\measuredangle(A, D, B)<\pi$ and $a=\measuredangle(C, B, A)$ and $b=\measuredangle(B, A, C)$ and $c=$ $\measuredangle(D, B, A)$ and $d=\measuredangle(C, A, D)$ and $R \cdot \cos s=\frac{\sin a}{\sin (a+b)}$ and $R \cdot \sin s=$ $\frac{\sin c}{\sin (b+d+c)}$ and $0<\vartheta<\pi$ and $\sin (2 \cdot s) \cdot \cos d=\cos (2 \cdot \vartheta)$. Then $|D-C|=$ $|A-B| \cdot \sqrt{2} \cdot R \cdot \sin \vartheta$.

Proof: $|D-C|^{2}=|A-B|^{2} \cdot\left((R \cdot \cos s)^{2}+(R \cdot \sin s)^{2}-2 \cdot(R \cdot \cos s) \cdot(R \cdot\right.$ $\sin s) \cdot \cos d) \cdot|D-C| \neq-|A-B| \cdot \sqrt{2} \cdot R \cdot \sin \vartheta$ by [13, (25)], [11, (42)].

(87) Suppose $A, C, B$ form a triangle and $\measuredangle(A, C, B)<\pi$ and $D, A, C$ form a triangle and $\measuredangle(A, D, C)=\frac{\pi}{2}$. Then $|D-C|=\left(\frac{|A-B| \cdot \sin \measuredangle(C, B, A)}{\sin (\measuredangle(B, A, C)+\measuredangle(C, B, A))}\right)$. $\sin \measuredangle(C, A, D)$. The theorem is a consequence of $(76)$.

(88) Suppose $B, C, A$ form a triangle and $\measuredangle(B, C, A)<\pi$ and $D, C, A$ form a triangle and $\measuredangle(C, D, A)=\frac{\pi}{2}$. Then $|D-C|=\left(\frac{|A-B| \cdot \sin \measuredangle(A, B, C)}{\sin (\measuredangle(A, B, C)+\measuredangle(C, A, B))}\right)$. $\sin \measuredangle(D, A, C)$. The theorem is a consequence of $(75)$.

(89) Suppose $A, C, B$ form a triangle and $\measuredangle(A, C, B)<\pi$ and $D, A, C$ form a triangle and $\measuredangle(A, D, C)=\frac{\pi}{2}$ and $A \in \mathcal{L}(B, D)$ and $A \neq D$. Then $|D-C|=$ $\left(\frac{|A-B| \cdot \sin \measuredangle(C, B, A)}{\sin (\measuredangle(C, A, D)-\measuredangle(C, B, A))}\right) \cdot \sin \measuredangle(C, A, D)$. The theorem is a consequence of (87).

(90) Suppose $B, C, A$ form a triangle and $\measuredangle(B, C, A)<\pi$ and $D, C, A$ form a triangle and $\measuredangle(C, D, A)=\frac{\pi}{2}$ and $A \in \mathcal{L}(D, B)$ and $A \neq D$. Then $|D-C|=\left(\frac{|A-B| \cdot \sin \measuredangle(A, B, C)}{\sin (\measuredangle(D, A, C)-\measuredangle(A, B, C))}\right) \cdot \sin \measuredangle(D, A, C)$.

Proof: $\sin (\measuredangle(C, A, B)+\measuredangle(A, B, C))=\sin (\measuredangle(D, A, C)-\measuredangle(A, B, C))$ by [4, (1)], [3, (8)].

\section{REFERENCES}

[1] Grzegorz Bancerek, Czesław Byliński, Adam Grabowski, Artur Korniłowicz, Roman Matuszewski, Adam Naumowicz, Karol Pąk, and Josef Urban. Mizar: State-of-the-art and beyond In Manfred Kerber, Jacques Carette, Cezary Kaliszyk, Florian Rabe, and Volker Sorge, editors, Intelligent Computer Mathematics, volume 9150 of Lecture Notes in Computer Science, pages 261-279. Springer International Publishing, 2015. ISBN 978-3319-20614-1. doi 10.1007/978-3-319-20615-8_17.

[2] R. Campbell. La trigonométrie. Que sais-je? Presses universitaires de France, 1956.

[3] Wenpai Chang, Yatsuka Nakamura, and Piotr Rudnicki. Inner products and angles of complex numbers. Formalized Mathematics, 11(3):275-280, 2003.

[4] Roland Coghetto. Some facts about trigonometry and Euclidean geometry. Formalized Mathematics, 22(4):313-319, 2014. doi 10.2478/forma-2014-0031.

[5] Roland Coghetto. Morley's trisector theorem. Formalized Mathematics, 23(2):75-79, 2015. doi $10.1515 /$ forma-2015-0007.

[6] Roland Coghetto. Circumcenter, circumcircle and centroid of a triangle. Formalized Mathematics, 24(1):19-29, 2016. doi 10.1515/forma-2016-0002.

[7] H.S.M. Coxeter and S.L. Greitzer. Geometry Revisited. The Mathematical Association of America (Inc.), 1967.

[8] Akihiro Kubo. Lines on planes in $n$-dimensional Euclidean spaces Formalized Mathematics, 13(3):389-397, 2005 . 
[9] Akihiro Kubo. Lines in $n$-dimensional Euclidean spaces. Formalized Mathematics, 11(4): $371-376,2003$.

[10] Akihiro Kubo and Yatsuka Nakamura. Angle and triangle in Euclidean topological space. Formalized Mathematics, 11(3):281-287, 2003.

[11] Marco Riccardi. Heron's formula and Ptolemy's theorem. Formalized Mathematics, 16 (2):97-101, 2008. doi 10.2478/v10037-008-0014-2

[12] Boris A. Shminke. Routh's, Menelaus' and generalized Ceva's theorems. Formalized Mathematics, 20(2):157-159, 2012. doi 10.2478/v10037-012-0018-9

[13] Andrzej Trybulec and Czesław Byliński. Some properties of real numbers Formalized Mathematics, 1(3):445-449, 1990.

[14] Yuguang Yang and Yasunari Shidama. Trigonometric functions and existence of circle ratio. Formalized Mathematics, 7(2):255-263, 1998.

Received December 30, 2015 